\title{
DISSEMINAÇÃO DA PESQUISA CIENTÍFICA NO ESPORTE DE ALTO RENDIMENTO NO BRASIL - ANÁLISE DO PERÍODO PRÉ- OLÍMPICO RIO 2016
}

Cacilda Mendes dos Santos Amaral

Doutora em Ciências

Universidade do Estado de Minas Gerais - Unidade Divinópolis cacilda.amaral@uemg.br

Flávia da Cunha Bastos

Livre Docente

Escola de Educação Física e Esporte da Universidade de São Paulo

flaviacb@usp.br

Maria Tereza Silveira Böhme

Professora Titular

Escola de Educação Física e Esporte da Universidade de São Paulo terbohme@gmail.com

\section{Resumo}

Objetivos: O objetivo do estudo foi identificar ações visando a disseminação de conteúdo científico voltados ao EAR para atletas e treinadores durante o período pré-olímpico do Jogos Rio 2016, nos níveis federal, estadual e municipal.

Metodologia/Abordagem: Estudo de abordagem qualitativa e análise documental, com base nos fatores críticos apresentados no modelo SPLISS relativos ao Pilar 9.

Originalidade/Relevância: $\mathrm{O}$ estudo aponta possíveis lacunas e promove o compartilhamento de boas práticas entre as esferas de governo, possibilitando melhor aplicação do conhecimento científicos produzido pela ciência do esporte na prática.

Principais Resultados: Os resultados mostram que no nível federal, tanto Ministério do Esporte quanto Comitê Olímpico do Brasil possuem Programas de disseminação da ciência, destaque para o Laboratório Olímpico mantido pelo Comitê Olímpico do Brasil. No nível estadual e municipal, poucos mantêm iniciativas. Foi possível observar uma sobreposição de ações desenvolvidas pelas organizações, além de uma desarticulação, evidenciando a falta de diretrizes nacionais para a disseminação da ciência do esporte.

Contribuições teórico-metodológicas: As implicações práticas deste estudo estão relacionadas à melhora dos processos de disseminação da ciência à treinadores e atletas e o fomento da discussão a respeito da responsabilidade dos entes envolvidos com o EAR.

Palavras-chave: Ciência. Disseminação de Informação. Esportes. Políticas Públicas.

\section{$\underline{\text { Cite como }}$}

American Psychological Association (APA)

Amaral, C. M. dos S., Bastos, F. da C., \& Böhme, M. T. S. . (2021, maio/ago.). Disseminação da pesquisa científica no esporte de alto rendimento no Brasil - análise do período Pré-olímpico Rio 2016. PODIUM Sport, Leisure and Tourism Review, São Paulo, 10(2), 1-23. https://doi.org/10.5585/podium.v10i2.16886. 
Amaral, C. M. dos S., Bastos, F. da C., \& Böhme, M. T. S. (2021, maio/ago.). Disseminação da pesquisa científica no esporte de alto rendimento no Brasil - análise do período Pré-olímpico Rio 2016

\title{
DISSEMINATION OF SCIENTIFIC RESEARCH IN HIGH-PERFORMANCE SPORT IN BRAZIL - ANALYSIS OF THE RIO 2016 PRE-OL YMPIC PERIOD
}

\begin{abstract}
Objectives: The aim of the study was to identify actions aimed at the dissemination of high performance sports scientific content for athletes and coaches during the pre-Olympic period of the Rio 2016 Games, at the federal, state and municipal levels.

Methodology / Approach: Qualitative approach study and document analysis, based on the critical factors presented in the SPLISS model for Pillar 9.

Originality / Relevance: The study points to possible gaps and promotes the sharing of good practices among the spheres of government, enabling a better application of the scientific knowledge produced by sports science in practice.

Main results: The results show that at the federal level, both the Ministry of Sports and the Brazilian Olympic Committee have Science dissemination programs, highlighting the Laboratório Olímpico, maintained by the Brazilian Olympic Committee. At the state and municipal levels, few maintain initiatives. It was possible to observe an overlap of actions developed by the organizations, in addition to a disarticulation, showing the lack of national guidelines for the dissemination of sports science.

Theoretical and methodological contributions: The practical implications of this study are related to the improvement of science dissemination processes to coaches and athletes and the promotion of discussion regarding the responsibility of the entities involved with the high performance sports.
\end{abstract}

Keywords: Science. Information Dissemination. Sports. Public Policies.

\section{DIFUSIÓN DE LA INVESTIGACIÓN CIENTÍFICA EN DEPORTES DE ALTO RENDIMIENTO EN BRASIL - ANÁLISIS DEL PERÍODO PREOLÍMPICO RÍO 2016}

\section{Resumen}

Objetivos: El objetivo del estudio fue identificar acciones encaminadas a la difusión de contenido científico orientado a los deportes de alto rendimiento para deportistas y entrenadores durante el período preolímpico de los Juegos Río 2016, a nivel federal, estatal y municipal.

Metodología / Enfoque: Estudio de enfoque cualitativo y análisis documental, basado en los factores críticos presentados en el modelo SPLISS para Pilar 9.

Originalidad / Relevancia: El estudio apunta a posibles lagunas y promueve el intercambio de buenas prácticas entre los ámbitos de gobierno, lo que permite una mejor aplicación del conocimiento científico producido por la ciencia del deporte en la práctica.

Resultados principales: Los resultados muestran que a nivel federal, tanto el Ministerio de Deportes como el Comité Olímpico Brasileño cuentan con programas de difusión de la Ciencia, destacando el Laboratorio Olímpico que mantiene el Comité Olímpico Brasileño. A nivel estatal y municipal, pocos mantienen iniciativas. Se pudo observar una superposición de acciones desarrolladas por las organizaciones, además de una desarticulación, evidenciando la falta de lineamientos nacionales para la difusión de la ciencia del deporte.

Contribuciones teóricas y metodológicas: Las implicaciones prácticas de este estudio están relacionadas con la mejora de los procesos de difusión de la ciencia a entrenadores y deportistas y la promoción de la discusión sobre la responsabilidad de las entidades involucradas con la EAR.

Palabras-clave: Ciencias. Diseminacion de informacion. Deportes. Políticas públicas. 
Amaral, C. M. dos S., Bastos, F. da C., \& Böhme, M. T. S. (2021, maio/ago.). Disseminação da pesquisa científica no esporte de alto rendimento no Brasil - análise do período Pré-olímpico Rio 2016

\section{Introdução}

Tanto a ciência do esporte como a interdisciplinaridade das áreas em prol do desenvolvimento de atletas começaram a ser foco de investimentos de alguns países há alguns anos atrás, muito estimulado por uma corrida ao sucesso esportivo, denominada "the global sporting arms race", em que investir estrategicamente no esporte de alto-rendimento passou a ser uma característica de países que possuem sucesso internacional no esporte (De Bosscher, Bingham, Shibli, Van Bottenburg \& De Knop, 2008). Inicialmente os investimentos se voltaram aos equipamentos e instalações esportivas, isto porque o investimento de ciência nestes ramos tende a gerar mais lucro do que pesquisas voltadas especificamente aos atletas, como estudos em nutrição, preparação física e psicológica (Houlihan \& Green, 2008).

A ciência do esporte é pensada essencialmente como pesquisa aplicada, usada para orientar a prática do esporte, fornecendo evidências de forma a melhorar o desempenho esportivo (Bishop, 2008; Bishop, Burnett, Farrow, Gabbett \& Newton, 2006). Entretanto, parece ser consenso de que a aplicação do conhecimento gerado pela ciência do esporte na prática ainda é deficitária e por vezes pouco relevante para treinadores e atletas (Bishop, 2008), e que há necessidade de comunicar os resultados das pesquisas para este público, e não restringir o conhecimento produzido pela ciência do esporte apenas à comunidade acadêmica (Bishop et al., 2006).

Em outras nações, a produção de conhecimento voltado ao esporte de alto rendimento (EAR) possui uma íntima relação com prática esportiva (Digel, 2002, 2005; Green \& Houlihan, 2006; Houlihan \& Green, 2008; Porto \& Gurgel, 2015), ou seja, o conhecimento produzido em laboratórios e Universidades é disseminado e utilizado efetivamente na prática do treinamento de atletas de alto rendimento, ou mesmo produzido especificamente para este fim.

No Brasil, ações governamentais de apoio a projetos de pesquisa e a implantação de cursos de pós-graduação a partir da década de 1970, proporcionaram um impulso para o desenvolvimento acadêmico e produção de conhecimento científico nas áreas de Educação Física e Esporte (Brasil, 1993; Tani, 1996). No entanto, ainda é verificada a necessidade de investimentos na formação de pesquisadores, ampliação dos programas de pós-graduação que direcionem suas pesquisas ao esporte, equilíbrio no desenvolvimento de áreas não valorizadas e que são estratégicas, e fortalecimento de pesquisas aplicadas voltadas ao desenvolvimento do esporte (Corrêa, Corrêa, \& Rigo, 2019; De Bosscher et al., 2015; Octaviano, 2010). 
Amaral, C. M. dos S., Bastos, F. da C., \& Böhme, M. T. S. (2021, maio/ago.). Disseminação da pesquisa científica no esporte de alto rendimento no Brasil - análise do período Pré-olímpico Rio 2016

Recentemente existiram ações voltadas a fornecer suporte financeiro ao desenvolvimento da ciência, tecnologia e inovação relacionadas ao esporte, decorrentes, principalmente da realização de megaeventos esportivos em território brasileiro (Terra, Batista, Almeida \& Campos, 2011). Entretanto, apesar de fundamentais para o desenvolvimento da ciência do esporte no Brasil, estas iniciativas ainda são pontuais e carecem de aperfeiçoamento no processo de seleção e avaliação dos projetos de pesquisa financiados e aperfeiçoamento no que tange à pertinência dos mesmos, dando prioridade a pesquisas centradas de fato no desenvolvimento da ciência do esporte e ao EAR (Viveiros, Moreira, Bishop \& Aoki, 2015).

Segundo Porto e Gurgel (2015), houve um abandono das políticas públicas que se utilizam de recursos de Universidades e de institutos de pesquisa para a realização de estudos voltados ao EAR. Pesquisadores sugerem que para o desenvolvimento da pesquisa voltada a esse segmento seria primordial a destinação de recursos e infraestrutura aos laboratórios, e a aproximação de instituições de ensino e pesquisa com as confederações e federações esportivas, com a finalidade de compreender a demanda de produção de conhecimento por parte dos envolvidos com a prática esportiva (Porto \& Gurgel, 2015). Este tipo de iniciativa poderia ser desenvolvido tanto em âmbito federal, como também nos estados e municípios brasileiros, principalmente aqueles que já contam com a presença de instituições de ensino e pesquisa em esporte, assim como laboratórios.

A ciência e inovação figuram entre os fatores importantes para o desenvolvimento do esporte em um país e, tanto no que diz respeito à questão de apoio tecnológico (equipamentos, instalações esportivas e vestuário), como também pesquisas voltadas à compreensão cada vez melhor do ambiente de treinamento, implementação dos resultados científicos na prática esportiva e resposta dos atletas de diferentes modalidades a variados tipos de treinamento físico, técnico e tático (Green \& Houlihan, 2006; Zysko, 2008).

Dado este panorama, o objetivo deste trabalho foi identificar ações visando a disseminação de conteúdo científico voltados ao EAR para atletas e treinadores durante o período pré-olímpico do Jogos Rio 2016, nos níveis federal, estadual e municipal. O propósito é apontar possíveis lacunas e promover o compartilhamento de boas práticas entre as esferas de governo, possibilitando melhor aplicação do conhecimento científicos produzido pela ciência do esporte na prática, tanto no treinamento como na competição de atletas de alto rendimento, colaborando para o estreitamento da relação e interação da ciência com a prática esportiva. 
Amaral, C. M. dos S., Bastos, F. da C., \& Böhme, M. T. S. (2021, maio/ago.). Disseminação da pesquisa científica no esporte de alto rendimento no Brasil - análise do período Pré-olímpico Rio 2016

\section{Revisão de literatura}

Sendo o resultado a essência do esporte de alto rendimento (Zysko, 2008), os fatores que determinam o sucesso no desenvolvimento de atletas de alto rendimento têm sido objeto de estudo de diversos autores (Böhme; Bastos, 2016; De Bosscher et al., 2006, 2015; Digel, 2005; Green; Oakley, 2001; Röger et al., 2010; Truyens et al., 2014). É possível verificar que há fatores em comum nos diversos modelos apresentados nestes estudos, como suporte financeiro, desenvolvimento e identificação do talento esportivo, instalações esportivas apropriadas, dentre outros.

Se o esporte de alto rendimento for entendido de forma sistêmica, e que seu desenvolvimento se dá, entre outros fatores, a partir da promoção de políticas públicas, a análise de como diferentes países organizam seus sistemas e políticas, pode ser considerado no estabelecimento de ações estratégicas (Mazzei et al., 2015). Houlihan e Green (2008) comparam diferentes modelos que elencam fatores que contribuem para o sucesso no esporte de alto rendimento, identificando três clusters distintos em que estes fatores podem ser organizados: Fatores relacionados ao contexto, ao processo e a fatores específicos do esporte de alto rendimento (Quadro 1).

Nenhum dos modelos criados pode explicar de fato o sucesso internacional no esporte, o que parece ser mais adequado a um país pode se apresentar falho para outro (De Bosscher et al., 2006), entretanto eles emanam alguns indicadores que podem ser considerados pelas nações em seus programas de esporte de alto rendimento. 
Amaral, C. M. dos S., Bastos, F. da C., \& Böhme, M. T. S. (2021, maio/ago.). Disseminação da pesquisa científica no esporte de alto rendimento no Brasil - análise do período Pré-olímpico Rio 2016

Quadro 1 - Modelos e os respectivos fatores que contribuem para o sucesso no esporte de alto rendimento

\begin{tabular}{|c|c|c|c|c|}
\hline Fatores & $\begin{array}{c}\text { Oakley e Green } \\
\text { (2001) }\end{array}$ & Digel (2002) & $\begin{array}{c}\text { Consórcio } \\
\text { SPLISS (2006) }\end{array}$ & $\begin{array}{c}\text { Green e } \\
\text { Houlihan (2005) }\end{array}$ \\
\hline \multirow[t]{3}{*}{ Contexto } & $\begin{array}{l}\text { Uma cultura } \\
\text { excelente }\end{array}$ & $\begin{array}{l}\text { Suporte público } \\
\text { (especialmente } \\
\text { financeiro) }\end{array}$ & Suporte financeiro & \multirow[t]{3}{*}{$\begin{array}{l}\text { Suporte para } \\
\text { atletas"tempo } \\
\text { integral" }\end{array}$} \\
\hline & Fundos apropriados & $\begin{array}{l}\text { Sucesso econômico } \\
\text { e negócios com } \\
\text { patrocinadores }\end{array}$ & $\begin{array}{l}\text { Participação no } \\
\text { esporte }\end{array}$ & \\
\hline & & $\begin{array}{l}\text { Suporte positivo da } \\
\text { mídia na cultura do } \\
\text { esporte }\end{array}$ & Pesquisa científica & \\
\hline \multirow[t]{5}{*}{ Processual } & $\begin{array}{l}\text { Papel claro das } \\
\text { diferentes entidades }\end{array}$ & \multirow{5}{*}{$\begin{array}{l}\text { Desenvolvimento } \\
\text { de talentos pelo } \\
\text { sistema } \\
\text { educacional } \\
\text { Desenvolvimento } \\
\text { de talentos pelas } \\
\text { forças armadas }\end{array}$} & $\begin{array}{l}\text { Sistema de } \\
\text { Identificação e } \\
\text { Desenvolvimento } \\
\text { de talento }\end{array}$ & \\
\hline & $\begin{array}{l}\text { Simplicidade na } \\
\text { administração }\end{array}$ & & $\begin{array}{l}\text { Suporte para } \\
\text { atletas e pós- } \\
\text { carreira }\end{array}$ & \\
\hline & $\begin{array}{l}\text { Sistema de } \\
\text { monitoramento do } \\
\text { progresso dos atletas }\end{array}$ & & $\begin{array}{l}\text { Abordagem } \\
\text { integrada para o } \\
\text { desenvolvimento } \\
\text { de políticas }\end{array}$ & \\
\hline & $\begin{array}{l}\text { Identificação de } \\
\text { talento e segmentação } \\
\text { dos recursos }\end{array}$ & & $\begin{array}{l}\text { Provisão e } \\
\text { desenvolvimento } \\
\text { de treinadores }\end{array}$ & \\
\hline & $\begin{array}{l}\text { Sistema de } \\
\text { planejamento para } \\
\text { cada modalidade } \\
\text { Suporte ao atleta }\end{array}$ & & & \\
\hline \multirow[t]{3}{*}{ Específico } & $\begin{array}{l}\text { Programas } \\
\text { competitivos bem } \\
\text { estruturados }\end{array}$ & \multirow[t]{3}{*}{$\begin{array}{l}\text { Serviço de suporte } \\
\text { da ciência do } \\
\text { esporte }\end{array}$} & $\begin{array}{l}\text { Competição } \\
\text { internacional }\end{array}$ & $\begin{array}{l}\text { Hierarquia de } \\
\text { oportunidade de } \\
\text { competição } \\
\text { centrara na } \\
\text { preparação para } \\
\text { eventos } \\
\text { internacionais }\end{array}$ \\
\hline & $\begin{array}{l}\text { Instalações } \\
\text { específicas bem } \\
\text { desenvolvidas }\end{array}$ & & $\begin{array}{l}\text { Instalações de } \\
\text { treino }\end{array}$ & $\begin{array}{l}\text { Desenvolvimento } \\
\text { de instalações para } \\
\text { o alto rendimento }\end{array}$ \\
\hline & & & & $\begin{array}{l}\text { Provisão de } \\
\text { treinadores, } \\
\text { ciência do esporte } \\
\text { e serviços de } \\
\text { suporte de } \\
\text { medicina } \\
\text { esportiva }\end{array}$ \\
\hline
\end{tabular}

Fonte: Adaptado de (HOULIHAN; GREEN, 2008).

A ciência e inovação figuram entre os fatores apresentados pelos modelos, tanto no que diz respeito à questão de apoio tecnológico (equipamentos, instalações esportivas e vestuário) como também pesquisas voltadas à compreensão cada vez melhor do ambiente de treinamento, implementação dos resultados científicos na prática esportiva e resposta dos atletas de 
Amaral, C. M. dos S., Bastos, F. da C., \& Böhme, M. T. S. (2021, maio/ago.). Disseminação da pesquisa científica no esporte de alto rendimento no Brasil - análise do período Pré-olímpico Rio 2016

diferentes modalidades a variados tipos de treinamento físico, técnico e tático (Green; Houlihan, 2006; Zysko, 2008).

Segundo Green e Houlihan (2006), tanto a ciência do esporte como a medicina em prol do desenvolvimento de atletas começaram a ser foco de investimentos com o advento de substancial verba pública direcionada ao esporte de alto rendimento. Inicialmente os investimentos se voltaram aos equipamentos e instalações esportivas, isto porque o investimento de ciência nestes ramos tende a gerar mais lucro do que pesquisas voltadas aos atletas, como estudos em nutrição, preparação física e psicológica (Houlihan; Green, 2008).

Adicionalmente, as modalidades não têm explorado a ciência do esporte em todo seu potencial, principalmente em relação à preparação e treinamento de atletas para competições (Green; Houlihan, 2006). Sendo uma área que ainda requer maior desenvolvimento para se converter em diferencial ou vantagem competitiva dos países (De Bosscher et al., 2008), a ciência do esporte tem despontado como um dos fatores que despertou maior interesse para investimentos na última década (De Bosscher et al., 2015).

$\mathrm{Na}$ China existem nove universidades e mais de 60 faculdades de esporte, além da existência de um Instituto Nacional de Ciências do Esporte, subordinado e coordenado diretamente pelo Ministério do Esporte (Houlihan; Green, 2008). Nesse país, há clara diretriz para que todos os pesquisadores que trabalham no Instituto sejam contratados em tempo integral e orientados a direcionar suas pesquisas ao treinamento de atletas de alto rendimento. Os acadêmicos e pesquisadores nas universidades também contribuem direta e indiretamente com o treinamento dos medalhistas de ouro nas áreas de técnicas de treinamento, psicologia esportiva, medicina esportiva, fisiologia, biomecânica e sociologia do esporte (Porto; Gurgel, 2015).

Nos Estados Unidos, a capacidade de pesquisa em ciência do esporte também está fortemente calcada nas universidades, que mantém relação direta com as equipes técnicas das modalidades de alto rendimento para difusão do conhecimento, apesar de não haver um sistema coordenado ou um instituto nacional (Houlihan; Green, 2008). Outros países optam por possuir um instituto ou centro de pesquisa específico para o esporte de alto rendimento. O modelo alemão é baseado na execução de projetos apoiados pelo Instituto Federal de Ciências do Esporte, que geralmente são implementados em cooperação com diferentes institutos de universidades e centros olímpicos de treinamento (Porto; Gurgel, 2015), e baseados em 4 pilares: a pesquisa básica, pesquisa orientada à aplicação, pesquisa orientada à prática e treinamento de treinadores (Houlihan; Green, 2008). 
Amaral, C. M. dos S., Bastos, F. da C., \& Böhme, M. T. S. (2021, maio/ago.). Disseminação da pesquisa científica no esporte de alto rendimento no Brasil - análise do período Pré-olímpico Rio 2016

Na Austrália, Digel (2005) menciona a centralização do controle do esporte no país pela Comissão Australiana de Esporte (ASC) e suas subdivisões distribuídas pelo território australiano e relação deste sistema com a ciência por meio do Instituto Australiano do Esporte (AIS), sendo indicado pelo autor como um dos melhores do mundo, dando direções para a pesquisa em ciência do esporte, e mais importante, a disseminação da ciência na prática (Green; Houlihan, 2006). Neste Instituto foram desenvolvidos 36 programas esportivos em 26 modalidades, indicando a estreita relação que o esporte possui com a ciência no país (Austrália, [s.d.]). Mesmo em países avaliados com uma baixa participação da ciência no desenvolvimento do esporte de alto rendimento, como Itália e Reino Unido, estes países possuem um instituto nacional de pesquisa voltada ao esporte de alto rendimento, a fim de desenvolver pesquisas específicas neste campo (Digel, 2002).

Já no Brasil, em estudo realizado a respeito do clima e ambiente de desenvolvimento do esporte de alto rendimento baseado no modelo Sports Policy Factors Leading to International Sporting Success (SPLISS), ao ser analizado o suporte e condução e disseminação da pesquisa científica em ciência do esporte, as autoras identificaram um cenário falho de comunicação entre as instituições que produzem o conhecimento e a prática efetica do treinamento de modalidades olímpicas, afetando assim a disseminação de conhecimento científico (Amaral; Bastos, 2016).

No modelo SPLISS, desenvolvido por um consórcio de pesquisadores a partir de extensiva revisão de literatura, análise de dados secundários e consulta com atletas e treinadores do esporte de alto rendimento, nove pilares são indicados como influenciadores do sucesso esportivo internacional (Figura 1) (De Bosscher et al., 2006). Este modelo foi amplamente discutido em âmbito acadêmico e aplicado em diversos países (Mazzei et al., 2015). Dentre os pilares, a "Pesquisa Científica e Inovação" é indicada como uma das áreas a serem desenvolvidas. 
Amaral, C. M. dos S., Bastos, F. da C., \& Böhme, M. T. S. (2021, maio/ago.). Disseminação da pesquisa científica no esporte de alto rendimento no Brasil - análise do período Pré-olímpico Rio 2016

Figura 1 - Representação do Modelo SPLISS e seus 9 pilares

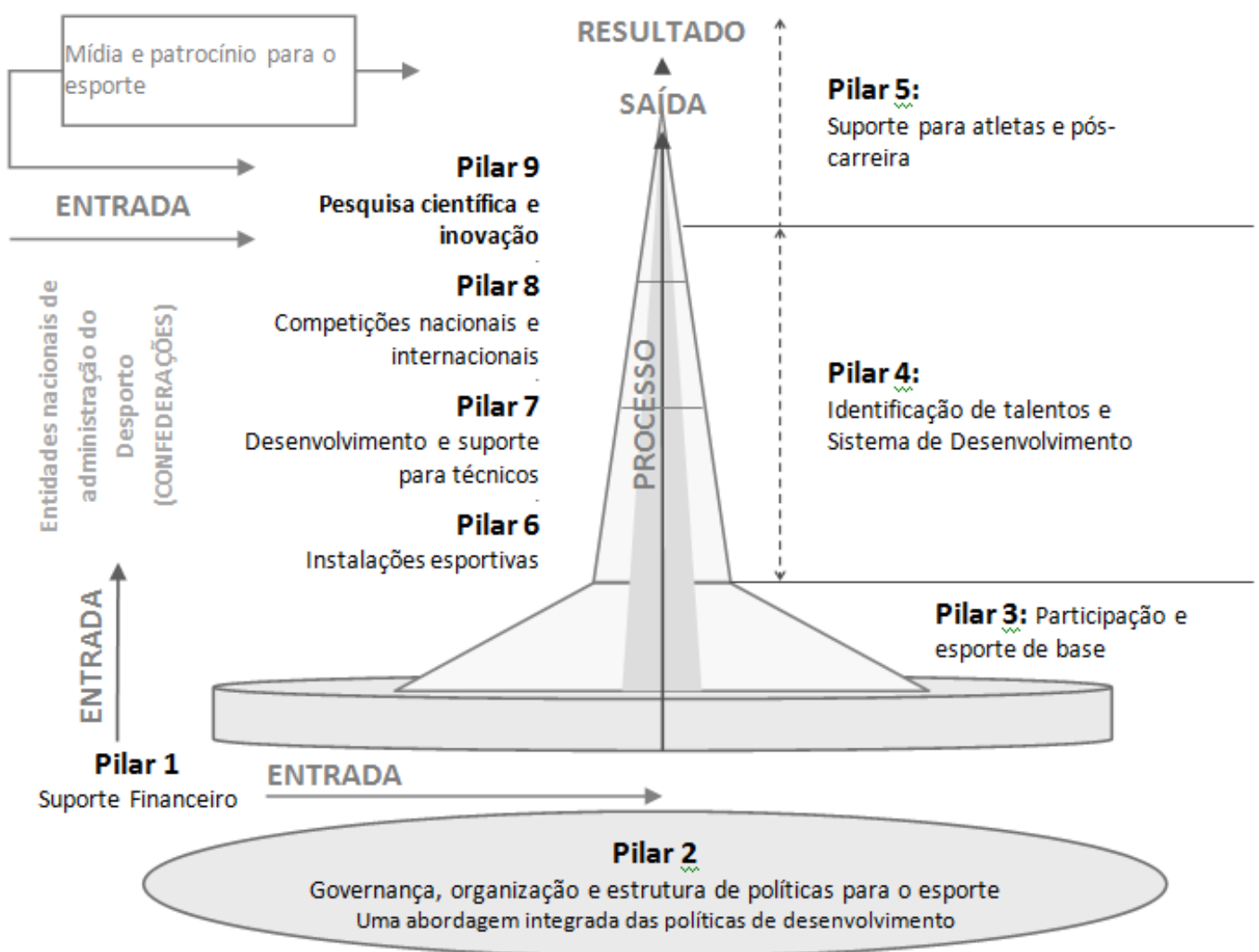

Fonte: Adaptado de DeBosscher et al. (2006).

Cada pilar é composto por indicadores e fatores críticos que devem ser desenvolvidos, sendo que o "Pilar 9" considera a contribuição científica para o esporte de alto rendimento e a disseminação da informação científica entre treinadores, atletas e dirigentes das modalidades olímpicas a partir de 10 fatores críticos de sucesso distribuídos em dois indicadores: a) Pesquisas científicas são conduzidas, coordenadas e disseminadas entre treinadores e confederações; b) O suporte da Ciência do Esporte é fornecido em cada um dos níveis de desenvolvimento do esporte de alto rendimento (De Bosscher et al., 2009; Böhme; Bastos, 2016).

Sendo objeto do estudo no "Pilar 9" a existência de um banco de dados atualizado regularmente de pesquisas científicas que pode ser consultado por treinadores a existência de uma rede para se comunicar e disseminar conteúdo científico para ligas, clubes, atletas e treinadores de alto rendimento (De Bosscher et al., 2009), optou-se pela utilização de dois fatores críticos de sucesso deste modelo para a avaliação da disseminação da ciência no esporte de alto rendimento no Brasil. 
Amaral, C. M. dos S., Bastos, F. da C., \& Böhme, M. T. S. (2021, maio/ago.). Disseminação da pesquisa científica no esporte de alto rendimento no Brasil - análise do período Pré-olímpico Rio 2016

\section{Método}

O estudo possui abordagem qualitativa, utilizando-se do método de análise documental (Queirós \& Graça, 2013; Pitts et al., 2018), tendo como unidade de análise o Esporte de Alto Rendimento no País.

Considerando que não há uma única organização no Brasil com responsabilidade específica pelo desenvolvimento do esporte de alto rendimento, e que atuam nesse segmento organizações governamentais e não-governamentais - nos níveis federal, estadual e municipal - (Meira, Bastos, \& Böhme, 2012), optou-se por abordar o máximo de entidades possíveis, sendo consideradas diferentes entidades para compor a amostra. A escolha da amostra de entidades selecionadas para a pesquisa foi determinadas pelos seguintes critérios: então Ministério do Esporte e Comitê Olímpico, por se tratarem das entidades a nível federal envolvidos com o desenvolvimento do EAR; Secretarias de esporte de nove estados (nível estadual) e suas respectivas capitais (nível municipal), e o Distrito Federal, definidos a partir de critérios como percentuais de atletas olímpicos que obtiveram medalhas nos Jogos Olímpicos de Londres em 2012; percentuais de atletas olímpicos que treinam no estado; percentuais dos orçamentos estaduais destinados ao Esporte de rendimento e percentuais de colaboração do estado para o PIB brasileiro.

A amostra final é detalhada no Quadro 2.

Quadro 2 - Entidades que tiveram seus documentos consultados

\begin{tabular}{|l|l|l|}
\hline \multicolumn{3}{|c|}{ Entidades Consultadas } \\
\hline \multicolumn{1}{|c|}{ Nível Federal } & \multicolumn{1}{|c|}{ Nível Estadual } & \multicolumn{1}{c|}{ Nível Municipal } \\
\hline - Ministério do & Secretarias Estaduais do & Secretarias Municipais de Esporte \\
Esporte; & Esporte de: & de: \\
- Comitê Olímpico & - São Paulo; & - São Paulo; \\
do Brasil & - Minas Gerais; & - Belo Horizonte; \\
& - Rio de Janeiro; & - Rio de Janeiro; \\
& - Paraná; & - Curitiba; \\
& - Rio Grande do Sul; & - Porto Alegre; \\
& - Santa Catarina; & - Florianópolis; \\
& - Distrito Federal; & - Salvador; \\
& - Bahia; & - Recife; \\
& - Pernambuco; & - Manaus. \\
& - Amazonas. & \\
\hline
\end{tabular}

Fonte: Elaborado pelas autoras. 
Amaral, C. M. dos S., Bastos, F. da C., \& Böhme, M. T. S. (2021, maio/ago.). Disseminação da pesquisa científica no esporte de alto rendimento no Brasil - análise do período Pré-olímpico Rio 2016

Os documentos consultados foram: publicações oficiais (revistas, encartes, informes, livros, relatórios, etc.) e sites e blogs das entidades previamente escolhidas, descriminados no Quadro 3. Foram selecionadas para análise todas as informações que apresentassem ações visando a disseminação da ciência voltada ao EAR, tais como bancos de dados de investigações científicas que pudessem ser consultados por treinadores e dirigentes, publicações e eventos científicos aos quais os treinadores e dirigentes tem acesso e acordos e parcerias com Universidades e Centros de Pesquisa para o desenvolvimento de estudos voltados ao esporte de alto rendimento.

Foi considerado período pré-olímpico o intervalo entre a última edição dos Jogos Olímpicos de Londres 2012 à realização dos Jogos Olímpicos Rio 2016. Sendo assim, as consultas foram realizadas entre 16 de julho de 2014 e 21 de fevereiro de 2016 . Foi realizada leitura crítica e análise dos documentos com objetivo de condensar a informação relacionada à disseminação da ciência no EAR de todos os documentos consultados e recolhidos (Queirós \& Graça, 2013), buscando informações que se relacionassem aos seguintes fatores críticos: há banco de dados de pesquisas científicas atualizado regularmente, que pode ser consultado por treinadores e confederações; e Há uma rede para se comunicar e disseminar conteúdo científico para confederações clubes, atléticas e treinadores de alto rendimento. 
Amaral, C. M. dos S., Bastos, F. da C., \& Böhme, M. T. S. (2021, maio/ago.). Disseminação da pesquisa científica no esporte de alto rendimento no Brasil - análise do período Pré-olímpico Rio 2016

Quadro 3 - Documentos analisados

\begin{tabular}{|c|c|}
\hline & Documentos consultados \\
\hline & Continua \\
\hline $\begin{array}{l}\text { Nível } \\
\text { Federal }\end{array}$ & 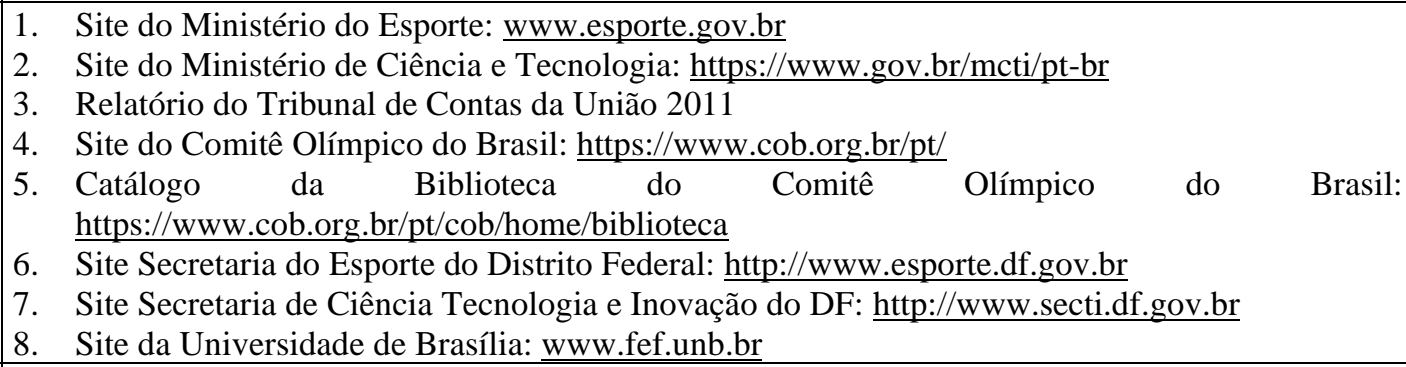 \\
\hline $\begin{array}{l}\text { Nível } \\
\text { Estadual }\end{array}$ & 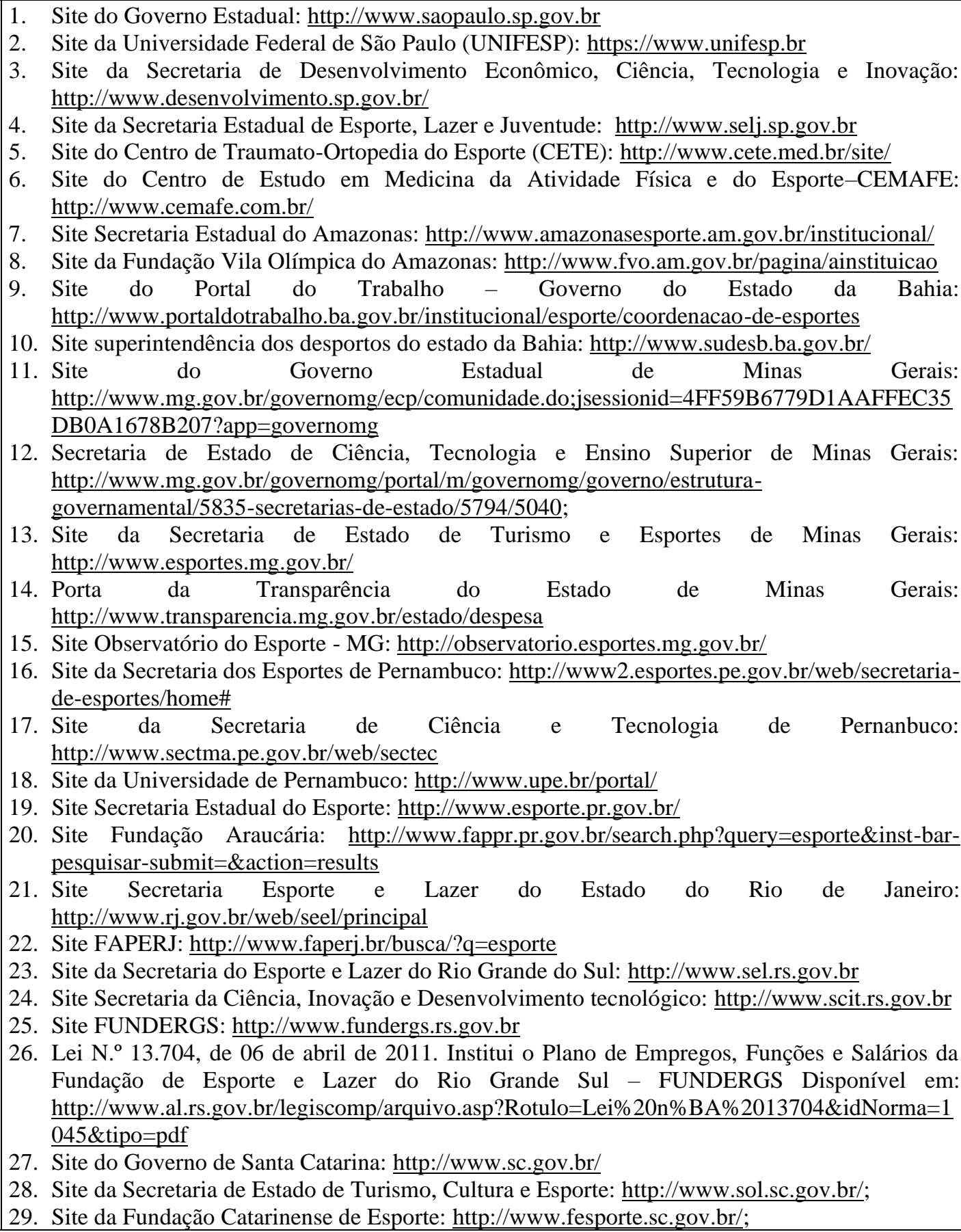 \\
\hline
\end{tabular}


Amaral, C. M. dos S., Bastos, F. da C., \& Böhme, M. T. S. (2021, maio/ago.). Disseminação da pesquisa científica no esporte de alto rendimento no Brasil - análise do período Pré-olímpico Rio 2016

\begin{tabular}{|c|c|}
\hline & $\begin{array}{l}\text { 30. Site do Conselho Estadual do Esporte: http://www.ced.sc.gov.br } \\
\text { 31. Centro de Ciências da Saúde e do Esporte: http://www.cefid.udesc.br/ }\end{array}$ \\
\hline & 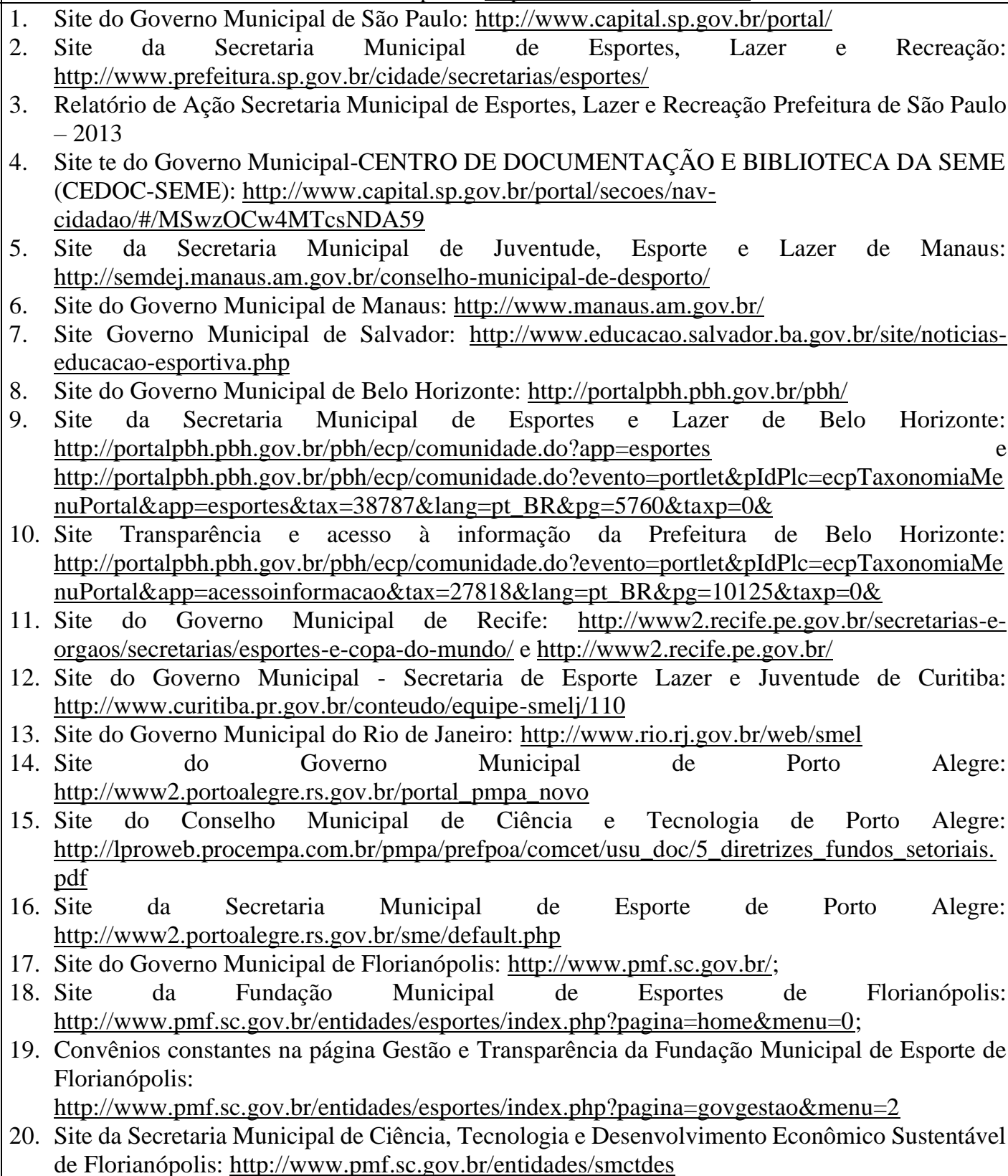 \\
\hline
\end{tabular}

Fonte: Elaboração própria.

\section{Resultados e discussão}

Semelhante a outros países, foram encontradas algumas ações desenvolvidas no âmbito do EAR no Brasil que visam a parceria com instituições de ensino superior com intuito de desenvolver e disseminar a ciência do esporte.

Em nível federal, foi identificada a Rede de Centros de Excelência Esportiva (Rede CENESP), programa do Ministério do Esporte, que atua de forma a coordenar ações em conjunto com Universidades, semelhante à encontrada em outros países como China e Estados 
Amaral, C. M. dos S., Bastos, F. da C., \& Böhme, M. T. S. (2021, maio/ago.). Disseminação da pesquisa científica no esporte de alto rendimento no Brasil - análise do período Pré-olímpico Rio 2016

Unidos (Houlihan; Green, 2008). Composto pelas estruturas físicas e administrativas, recursos humanos e materiais existentes em instituições de ensino superior, onde os centros ou núcleos estão implantados, cada CENESP é formado em estreita parceria com a Secretaria Nacional de Esporte de Alto Rendimento (SNEAR), o Comitê Olímpico do Brasil (COB), o Comitê Paraolímpico Brasileiro, com as entidades de administração do desporto em nível local, estadual e nacional, e com a iniciativa privada. Os Centros e os Núcleos da Rede são 9, e incluem Universidades Federais e Estaduais de 7 estados diferentes.

No plano plurianual da Rede CENESP dos anos de 2000-2003, apresentam-se três Programas que possuem ações relacionadas à disseminação da ciência. O primeiro deles, denominado "Programa Brasil Potência Esportiva", incluía Avaliação de Atletas de Rendimento, prevendo o acompanhamento de desempenho e dos resultados em competições nacionais e internacionais e a detecção de talentos esportivos. O segundo, denominado "Programa Esporte Direito de Todos", apresenta a ação de Promoção de Eventos e Intercâmbios Científicos e Tecnológicos para o Esporte com o objetivo principal de agregar profissionais e acadêmicos do esporte e de outras áreas correlatas com intuito de aproximar a prática e o conhecimento científico, a fim de promover o desenvolvimento esportivo.

Já no "Programa Gestão das Políticas de Esporte e Turismo", foi planejada uma ação de apoio a pesquisas científicas e tecnológicas na área do esporte com a intenção de possibilitar maior relação entre pesquisa, ensino e extensão universitária. A ação que leva o nome Estudos e Pesquisas Científicas e Tecnológicas para o Desenvolvimento do Esporte se propunha a estimular e apoiar a produção acadêmica, equipar laboratórios em parceria com agências de fomento. O mesmo programa também previa a ação de Edição e Distribuição de Material Técnico-didático do Esporte com objetivo de viabilizar o acesso a pesquisas científicas na área do esporte, publicando livros de ordem técnico-científicos-cultural e a promoção de publicações em periódicos com intuito de incentivar a divulgação de trabalhos acadêmicos.

Apesar do aparente esforço do governo federal em disseminar o conhecimento científico de forma que ele dê suporte a prática, não foram identificados programas em que atletas de alto rendimento e treinadores recebam apoio financeiro diretamente para serviços de apoio científico, como para a participação em congressos, o estudo em centros de pesquisa nacionais e internacionais, ou mesmo para a realização de pesquisas. Estes dados corroboram com a opinião de atletas e treinadores que avaliam como insuficiente o apoio recebido para estes fins (Amaral; Bastos, 2016). 
Amaral, C. M. dos S., Bastos, F. da C., \& Böhme, M. T. S. (2021, maio/ago.). Disseminação da pesquisa científica no esporte de alto rendimento no Brasil - análise do período Pré-olímpico Rio 2016

Ainda a nível federal, o COB mantém algumas iniciativas voltadas à difusão da ciência por meio do Instituto Olímpico Brasileiro (IOB), que tem como objetivo a promoção de intercâmbio e difusão de conhecimento a partir de eventos científicos e a manutenção de uma base de dados de informações relacionadas à ciência do esporte. A base de dados do COB é mantida pela Biblioteca do Comitê Olímpico, criada em 1996 possuindo um acervo físico e online relacionado aos Jogos e Movimento Olímpico, Jogos Pan-americanos, Jogos Sulamericanos, história e evolução do esporte no Brasil e no mundo. Aberto ao público em geral, este banco não aborda especificamente o EAR, mas apresenta-se como possibilidade de suporte a treinadores e dirigentes.

O conhecimento produzido por outras instituições, do país e do exterior, é difundido pelo COB através da revista "Laboratório Olímpico". De tiragem bimestral, a publicação tem o intuito de fornecer conhecimento a treinadores e atletas sobre pesquisas com aplicabilidade no EAR, com uma secção específica sobre a pesquisa científica e inovação/publicações de relatórios científicos dedicados exclusivamente ao segmento.

No nível estadual, o governo do Rio Grande do Sul (RS) possui um acordo entre Secretaria de Esporte e Lazer e a Universidade Federal do Rio Grande do Sul (UFRGS) no intuito de troca de informações técnicas e no desenvolvimento de projetos e estudos de forma integrada, no entanto não foram divulgadas maiores informações a respeito do acordo e de quais projetos estão sendo desenvolvidos de fato.

No Paraná (PR), o projeto de pesquisa "Projeto Genômica Esportiva” é resultante de um acordo entre governo estadual e Universidade Estadual de Londrina (UEL), ainda em conjunto com a Coordenação de Aperfeiçoamento de Pessoal de Nível Superior (CAPES) e iniciativa privada. Apesar de não se tratar de um projeto que vise o desenvolvimento de pesquisas exclusivamente voltadas ao EAR, apresenta-se como uma iniciativa do governo estadual em estabelecer parcerias visando o desenvolvimento científico do esporte no estado.

No Distrito Federal (DF), há projeto desenvolvido junto à Universidade de Brasília (UnB) no Centro de Excelência em Saltos Ornamentais, mas faltam informações a respeito da pesquisa e seus desdobramentos. Este projeto tem como objetivo o desenvolvimento da modalidade e de pesquisas científicas relacionadas ao treinamento esportivo de alto nível. Para os demais estados pesquisados, não parece haver acordos formais com Universidades.

Também foi possível verificar que nenhum estado apresenta uma via de comunicação e disseminação formalizada para veicular conteúdo científico entre seus treinadores, federações e organizações esportivas em geral, mesmo aqueles que possuem parcerias com universidade, 
Amaral, C. M. dos S., Bastos, F. da C., \& Böhme, M. T. S. (2021, maio/ago.). Disseminação da pesquisa científica no esporte de alto rendimento no Brasil - análise do período Pré-olímpico Rio 2016

ou mantêm projetos de desenvolvimento científico. Contanto com diversas entidades que atuam no desenvolvimento do esporte de alto rendimento no Brasil, seria interessante que as ações fossem realizadas de forma coordenada entre nível federal, estadual e municipal, de forma que o conhecimento produzido a partir de parcerias à nível estadual, por exemplo, pudesse ser divulgado nos canais de comunicação já estabelecidos pelas entidades de nível federal.

Quanto aos municípios estudados, apenas São Paulo apresenta uma iniciativa na disseminação da ciência do esporte, colocando à disposição do público geral no site oficial da Secretaria Municipal de Esportes, Lazer e Recreação (SEME), por meio do Centro de Documentação e Biblioteca da SEME, algumas publicações científicas, além de manter uma biblioteca física que também pode ser consultada livremente. Infelizmente o banco de pesquisas online está desatualizado há alguns anos, nos levando crer que o mesmo não é atualizado regularmente. Ainda assim, iniciativas como esta do Centro de Documentação e Biblioteca da SEME são um exemplo de como estruturar e organizar a informação/conhecimento para a utilização não só de treinadores e atletas, como de toda a comunidade.

Os resultados provenientes da análise dos documentos foram condensados e podem sem consultados no Quadro 4.

Quadro 4 - Resultados sintetizados da análise de documentos indiretos

\begin{tabular}{|c|c|c|}
\hline & Organizações Governamentais & Organizações Não-Governamentais \\
\hline Nível Federal & $\begin{array}{l}\text { Ministério do Esporte - Rede CENESP } \\
\text { - Avaliação de Atletas de Rendimento; } \\
\text { - Promoção de Eventos e Intercâmbios } \\
\text { Científicos e Tecnológicos para o } \\
\text { Esporte; } \\
\text { - Estudos e Pesquisas Científicas e } \\
\text { Tecnológicas para o } \\
\text { Desenvolvimento do Esporte; } \\
\text { - Edição e Distribuição de Material } \\
\text { Técnico-didático do Esporte. }\end{array}$ & $\begin{array}{l}\text { Comitê Olímpico do Brasil - Instituto } \\
\text { Olímpico Brasileiro; } \\
\text { - Realização de Eventos Científicos; } \\
\text { - Biblioteca do COB } \\
\text { - Revista Laboratório Olímpico. }\end{array}$ \\
\hline Nível Estadual & $\begin{array}{l}\text { RS - Parceira entre Secretaria de } \\
\text { esporte e UFRGS; } \\
\text { - PR - "Projeto Genômica Esportiva" } \\
\text { em parceria com UEL; } \\
\text { - DF - Centro de Excelência em Saltos } \\
\text { Ornamentais parceria com UnB; }\end{array}$ & \\
\hline Nível Municipal & $\begin{array}{l}\text { - São Paulo - Mantém Centro de } \\
\text { Documentação e Biblioteca da SEME }\end{array}$ & \\
\hline
\end{tabular}

Fonte: Elaborado pelas autoras.

Após a análise dos documentos, observa-se que a área de ciência do esporte voltada ao alto rendimento ainda pode ser amplamente explorada. Foi possível verificar, tal como indicado 
Amaral, C. M. dos S., Bastos, F. da C., \& Böhme, M. T. S. (2021, maio/ago.). Disseminação da pesquisa científica no esporte de alto rendimento no Brasil - análise do período Pré-olímpico Rio 2016

por Meira, Bastos e Böhme (2012), que as ações das organizações são desconexas e algumas vezes há sobreposições. Isto ocorre em parte por não haver responsabilidade específica, entre as organizações ligadas ao alto rendimento, pelo desenvolvimento e disseminação da ciência, podendo acarretar numa dificuldade de acesso às informações, demostrando a falta de sinergia entre os órgãos, diferente de alguns países como Austrália e Alemanha, que possuem seus processos mais unificados (Digel, 2005; Porto; Gurgel, 2015).

Em termos de ação governamental referente à Ciência, Tecnologia e Inovação para o Esporte no Brasil, foi criada em 1998 a Rede CENESP sob as diretrizes do Ministério do Esporte, apoiando o desenvolvimento do esporte por meio de pesquisas e serviços às principais equipes e atletas do país através de parceria com Universidades (Brasil, 2010) assim como realizados em países como China e Estados Unidos (Houlihan \& Green, 2008). Para além da amplitude de ações propostas pelo programa Rede CENESP, as informações do mesmo se encontram desatualizadas, não sendo possível uma confirmação de quais ações estão sendo de fato desenvolvidas.

No Balanço de Governo 2003-2010 já eram apresentados alguns questionamentos quanto ao impacto das ações promovidas pela Rede CENESP nos resultados esportivos dos atletas de alto rendimento, e a existência de uma possível lacuna na relação entre os estudos e pesquisas produzidos por meio de ações da rede e a formação de atletas (Brasil, 2010).

Colaborando para este diagnóstico, no relatório do Tribunal de Contas da União a respeito do EAR no Brasil, há indicações de um desconhecimento por parte de clubes e federações das ações e programas promovidos pela Rede CENESP (Brasil - Tribunal de Contas da União, 2011). Neste mesmo relatório há informações de que no ano de 2010 houve interrupção do repasse de verbas da SNEAR para a Rede CENESP, possivelmente ocasionando a paralisação das ações e por este motivo as informações do Programa estão desatualizadas no site do então Ministério do Esporte, que ainda assim indica o Programa como ativo.

No Programa Rede CENESP foi possível observar que as atividades correlatas à pesquisa são amplas, desde a instalação de centros de excelência em parceria com Universidades, até a edição de conteúdo científico. Esta última ação, apesar de importante, já é realizada também pelo COB na revista Laboratório Olímpico, que possui reconhecimento dos treinadores, uma vez que este é o único canal de disseminação citado pelos mesmo em pesquisa realizada (Amaral \& Bastos, 2016). Além da falta de informações acerca de material editado pelo programa do então Ministério do Esporte, o não reconhecimento deste tipo de material por 
Amaral, C. M. dos S., Bastos, F. da C., \& Böhme, M. T. S. (2021, maio/ago.). Disseminação da pesquisa científica no esporte de alto rendimento no Brasil - análise do período Pré-olímpico Rio 2016

parte dos treinadores verificado por Amaral e Bastos (2016) nos leva a crer que a ação não vem sendo desenvolvida nos últimos anos.

Ainda sobre a publicação do COB, Laboratório Olímpico, a ação se assemelha à realizada na pela entidade responsável pelo desenvolvimento do esporte de alto rendimento na Noruega (Olympiatoppen), que publica brochuras com conteúdo voltado a aspectos gerais do treinamento e nutrição de atletas (Houlihan \& Green, 2008).

A promoção de evento e intercâmbio científico também são ações propostas pelas duas esferas ligadas ao nível federal, ou seja, tanto o Ministério do Esporte quanto COB se propunham a realizar este tipo de ação. Entretanto, conforme apontado por Amaral e Bastos (2016), a maioria dos treinadores afirmam não ter participado deste tipo de evento nos últimos 12 meses promovidos por quaisquer organizações à que eles estão ligados (incluindo confederações e federações).

Quando comparado com outros países, o Brasil, apesar de possuir na Rede CENESP uma tentativa de articulação entre Universidades e o EAR, não possui um órgão ou instituo central voltado à pesquisa, pois a Rede CENESP não se configura desta forma nem trabalha de forma integrada (Amaral \& Bastos, 2016), como acontece em países como Alemanha, Austrália, Polônia e França (Digel, 2002). A inexistência de um centro de pesquisa nacional acaba dificultando o entendimento da demanda da prática para com a ciência, da definição de diretrizes para a realização de pesquisas e a própria transferência de conhecimento para treinadores, evidenciada na avaliação baixa que atletas e treinadores possuem a respeito da aplicabilidade da ciência em suas modalidades.

Pode-se verificar nas iniciativas dos governos estaduais de RS, PR e DF que as ações se assemelham em parte com a proposta da Rede CENESP do governo federal. A realização de parcerias com Universidades visa o desenvolvimento científico voltado ao esporte, mas é importante destacar que os projetos tanto de RS e PR não são voltados exclusivamente para o EAR, e o DF não apresenta dados claros sobre esta parceria. Também não foram encontrados dados que analisem criticamente e discutam qual é o impacto real dessas parcerias no esporte.

No nível municipal, há indícios de que os municípios têm dificuldade não só em estruturar a pesquisa dentro de sua organização, como também de difundi-la, uma vez que não foi encontrada nenhuma iniciativa municipal no que concerne à organização de um banco de dados atualizado com estudos que poderiam servir para que treinadores pudessem se aprimorar e buscar novos conhecimentos. 
Amaral, C. M. dos S., Bastos, F. da C., \& Böhme, M. T. S. (2021, maio/ago.). Disseminação da pesquisa científica no esporte de alto rendimento no Brasil - análise do período Pré-olímpico Rio 2016

Com a falta de um banco de dados com material científico atualizado, ficam prejudicadas quaisquer ações que queiram disseminar conteúdo científico esportivo entre os interessados. Como citado anteriormente, apenas São Paulo teve uma iniciativa neste sentido, com Centro de Documentação e Biblioteca da SEME, mas este banco de publicações não parece ser atualizado constante, uma vez que o acervo online possui apenas publicações antigas. Neste ponto fica prejudicada a utilização da ciência por parte dos treinadores em suas atividades de treinamento, uma vez que eles não possuem canais estabelecidos para obter este tipo de conhecimento. Ressalta-se também similaridade desta ação municipal com a ação desempenhada pelo $\mathrm{COB}$ em sua Biblioteca, que também possui acervo digital e físico.

Esta sobreposição de ações e a insuficiência na disseminação de conteúdo científico declarada principalmente por treinadores e dirigentes (Amaral \& Bastos, 2016) colabora com a visão de que há uma falta de coordenação e articulação no País no que tange a ciência do esporte. Essa visão foi apontada pelas autoras, revelando-se através da inexistência de um centro de pesquisa nacional, em colaboração com o fato de não haver responsabilidade específica entre as organizações envolvidas com o desenvolvimento do EAR quanto ao apoio da ciência.

A falta de coordenação no País é reafirmada por Mazzei et al. (2015), que consideram que, se por um lado é dada autonomia de gestão às organizações ligadas ao EAR, por outro lado, a falta de uma orientação central mínima quanto à responsabilidade de cada entidade, acaba por prejudicar a elaboração e aplicação coerente das políticas do esporte, o que acontece em nações com sucesso esportivo no âmbito internacional.

\section{Conclusão}

Por meio deste estudo, buscou-se traçar um panorama dos canais de disseminação da ciência voltada ao EAR no Brasil. Foi possível observar lacunas importantes no que tange o acesso de treinadores e dirigentes à ciência do esporte, mas também foi possível identificar boas práticas que poderiam ser incorporadas na prática de um maior número de treinadores. A partir da análise dos canais de disseminação de conteúdo científico para o EAR, pode-se verificar que o modo como o esporte está estruturado no Brasil, com a participação de organizações governamentais e não-governamentais que não possuem uma responsabilidade específica para o desenvolvimento do esporte, acaba por influenciar o modo como ações e programas que visam a disseminação da ciência no EAR ocorra. Além da sobreposição de ações, é possível verificar a falta de responsabilidade específica no esporte para a divulgação de conhecimento científico 
Amaral, C. M. dos S., Bastos, F. da C., \& Böhme, M. T. S. (2021, maio/ago.). Disseminação da pesquisa científica no esporte de alto rendimento no Brasil - análise do período Pré-olímpico Rio 2016

entre treinadores e uma desarticulação entre as entidades, que não mantém nenhuma ação em conjunto.

Mesmo para os Programas existentes, há falta de informações claras e atualizações sobre as ações realizadas pelos Programas, e em alguns casos é possível identificar a descontinuidade das ações. Experiências realizadas em outros países ou mesmo programas de organizações brasileiras podem servir de exemplo para que as entidades passem a se organizar de forma que o conhecimento científico chegue à prática de treinamento de atletas. A colaboração com Programas já existentes também é uma possibilidade, ou a divulgação de ações de entidades federais nos níveis estaduais e municipais também pode ser realizada.

Além disso, o convênio com Centros de Pesquisa e Universidades tem se mostrado, tanto no Brasil como em outros países, uma alternativa que beneficia ambos os lados e estreita os laços entre academia e campo prático. A inclusão de laboratórios nos centros de treinamento de atletas com a colaboração de pesquisadores é uma possibilidade para a inserção da ciência no dia a dia dos treinamentos de atletas.

Este estudo apresenta algumas limitações, principalmente no que tange a ordem metodológica. Sendo o estudo de um caso complexo, envolvendo muitas organizações que estão diretamente envolvidas com o desenvolvimento do EAR, não foi possível consultar todas as organizações. Também foi restrito o acesso a informações oficiais de algumas entidades, além de publicações desatualizadas das organizações estudadas. Temos que levar em consideração também que o estudo se baseou no modelo SPLISS e por este motivo ações importantes que foram desenvolvidas no âmbito da disseminação da ciência no EAR podem não ter sido consideradas por não serem consideradas por este modelo.

As implicações práticas deste estudo estão relacionadas à melhora dos processos de disseminação da ciência à treinadores e atletas através das entidades que administram o EAR no Brasil a partir de parcerias e estabelecimento de canais de comunicação oficiais entre as instituições e entre instituições e público alvo. Quanto às implicações teóricas, poderá fomentar a discussão a respeito da responsabilidade dos entes envolvidos com o EAR, e servir de base para a discussão de um sistema de produção de conhecimento em ciência do esporte e sua difusão entre os maiores interessados.

Para futuros estudos, indicamos a abordagem das possíveis ações de disseminação da ciência realizadas pelas entidades nas quais a maioria dos treinadores possui vínculo, como Clubes, Federações e Confederações de diferentes modalidades (sendo possível dessa forma a comparação entre modalidades). 
Amaral, C. M. dos S., Bastos, F. da C., \& Böhme, M. T. S. (2021, maio/ago.). Disseminação da pesquisa científica no esporte de alto rendimento no Brasil - análise do período Pré-olímpico Rio 2016

\section{Referências}

Amaral, C. M. S., \& Bastos, F. C. (2016). Pesquisa Científica e Inovação. In: Böhme , M. T. S. \& Bastos, F. C. (Eds.), Esporte de alto rendimento: fatores críticos, gestão e identificação de talentos (1 ${ }^{\mathrm{a}}$, pp. 204-226). São Paulo: Phorte Editora.

Austrália. (n.d.). Australian Institute of Sport. Australian Sports Commission. Australian Governament. http://www.ausport.gov.au/ais/about

Bishop, D. (2008). An Applied Research Model for the Sport Sciences. Sports Med, 38(3), 253-263.

Bishop, D., Burnett, A., Farrow, D., Gabbett, T., \& Newton, R. (2006). Sports Science Roundtable: Does Sports Science Research Influence Practice? International Journal of Sports Physiology and Performance, 1, 161-168. https://doi.org/10.1123/ijspp.1.2.161

Böhme, M. T. S., \& Bastos, F. C. (2016). Esporte de alto rendimento: fatores críticos, gestão e identificação de talentos, $1^{\mathrm{a},}$ São Paulo: Phorte Editora.

Brasil. (1993). Lei ${ }^{\circ}$ 8.672, de 6 de julho de 1993 - Institui normas gerais sobre o desporto e dá outras providências. Brasília, DF: Diário Oficial da República Federativa do Brasil.

Brasil. (2010). Balanço de Governo. Retrieved from:

http://www.balancodegoverno.presidencia.gov.br. Access: 23 nov. 2019.

Brasil - Tribunal de Contas da União. (2011). Esporte de Alto Rendimento / Tribunal de Contas da União. (A. Sherman, Ed.). Brasília: TCU, Secretaria de Fiscalização e Avaliação de Programas de Governo. Retrieved from:

http://portal2.tcu.gov.br/portal/page/portal/TCU/comunidades/programas_governo/areas _atuacao/esportes/Relatório_Esporte Alto Rendimento_Miolo.pdf. Access: 11 fev 2015.

Corrêa, M. R. D., Corrêa, L. Q., \& Rigo, L. C. (2019). A pós-graduação na educação física brasileira: condições e possibilidades das subáreas sociocultural e pedagógica. Revista Brasileira de Ciencias Do Esporte, 41(4), 359-366.

https://doi.org/10.1016/j.rbce.2018.03.009.

De Bosscher, V., Bingham, J., Shibli, S., Van Bottenburg, M., \& De Knop, P. (2008). The global Sporting Arms Race. An international comparative study on sports policy factors leading to international sporting success. Aachen: Meyer \& Meyer.

De Bosscher, V., De Knop, P., Van Bottenburg, M., \& Shibli, S. (2006). A Conceptual Framework for Analysing Sports Policy Factors Leading to International Sporting Success. European Sport Management Quarterly, 6(2), 185-215.

De Bosscher, V., De Knop, P., van Bottenburg, M., Shibli, S., \& Bingham, J. (2009). Explaining international sporting success: An international comparison of elite sport systems and policies in six countries. Sport Management Review, 12(3), 113-136. https://doi.org/10.1016/j.smr.2009.01.001.

De Bosscher, V., Shibli, S., Westerbeek, H., \& Van Bottenburg, M. (2015). Successful Elite 
Amaral, C. M. dos S., Bastos, F. da C., \& Böhme, M. T. S. (2021, maio/ago.). Disseminação da pesquisa científica no esporte de alto rendimento no Brasil - análise do período Pré-olímpico Rio 2016

Sport Policies: An internacional comparison of the Sports Policy factors Leading to Internacional Sporting Success (SPLISS 2.0) in 15 nations, 1 $^{\text {a }}$, Maidenhead: Meyer \& Meyer Sport.

Digel, H. (2002). A comparison of competitive sport systems. New Studies in Athletics, 17(1), $37-50$.

Digel, H. (2005). Comparison of successful sport systems. New Studies in Athletics, 20(2), 718.

Green, M., \& Houlihan, B. (2006). Elite Sport Development: Policy learning and political prioritie, $2^{\mathrm{a}}$, London and New York: Routledge Taylor \& Francis Group.

Green, M., \& Oakley, B. (2001). Elite sport development systems and playing to win: uniformity and diversity in international approaches. Leisure Studies, 20(4), 247-267. https://doi.org/10.1080/02614360110103598.

Houlihan, B., \& Green, M. (2008). Comparative Elite Sport Development: Systems, Structures and public policy, $1^{\text {a }}$, Burlington: Elsevier.

Mazzei, L. C., Meira, T. B., Bastos, F. C., Bohme, M. T. S., \& De Bosscher, V. (2015). High performance sport in Brazil Structure and policies comparison with the international context. Gestión y Política Pública, Volumen Te, 83-111.

Meira, T. B., Bastos, F. C., \& Böhme, M. T. S. (2012). Análise da estrutura organizacional do esporte de rendimento no Brasil: um estudo preliminar. Revista Brasileira de Educação Física e Esporte, 26(2), 251-262.

Octaviano, C. (2010). Especialista critica baixo investimento em pesquisas esportivas no país. Retrieved from:

http://www.comciencia.br/comciencia/handler.php?section=9\&reportagem=666. Access: 15 mar 2016.

Pitts, B. G., Li, M., \& Kim, A. (2018). Research Methods in Sport Management (2nd ed.). FIT Publishing.

Porto, F., \& Gurgel, J. L. (2015). Otimização Operacional dos Laboratórios de Pesquisa em Ciências do Esporte Pós Jogos Olímpicos de 2016 no Rio de Janeiro. In: Deslandes, A., DaCosta, L. P. \& Miragaya, A. (Eds.), O Futuro dos Megaeventos Esportivos $1^{\text {a }}$ Ediç̧ão, p. 496, Rio de Janeiro: FAPERJ.

Queirós, P., \& Graça, A. (2013). Análise de Conteúdo (enquanto técnica da informação no âmbito da investigação qualitativa). In: Mesquisa, I. \& Graça, A. (Eds.), Investigação Qualitativa em Desporto - Vl. II, $1^{\text {a }}$ Ed, Porto: Gráfica Maiadouro.

Röger, U., Rütten, A., Ziemainz, H., \& Hill, R. (2010). Quality of talent development systems: results from an international study. European Journal for Sport and Society, 7(1), 7-19. 
Amaral, C. M. dos S., Bastos, F. da C., \& Böhme, M. T. S. (2021, maio/ago.). Disseminação da pesquisa científica no esporte de alto rendimento no Brasil - análise do período Pré-olímpico Rio 2016

Tani, G. (1996). Vivências práticas no curso de graduação em educação física: necessidade, luxo ou perda de tempo? Caderno Documentos, (2), 1-22.

Terra, B., Batista, L. A., Almeida, M., \& Campos, S. R. C. (2011). A oportunidade de inovação no esporte é nossa! Polêm! Ca, 10(1), 74-90.

Truyens, J., De Bosscher, V., Heyndels, B., \& Westerbeek, H. (2014). A resource-based perspective on countries' competitive advantage in elite athletics. International Journal of Sport Policy and Politics, 6(3), 459-489.

https://doi.org/10.1080/19406940.2013.839954.

Viveiros, L., Moreira, A., Bishop, D., \& Aoki, M. S. (2015). Ciência do Esporte no Brasil: reflexões sobre o desenvolvimento das pesquisas, o cenário atual e as perspectivas futuras. Revista Brasileira de Educação Física e Esporte, 29(1), 163-175.

Zysko, J. (2008). Poland. In: Houlihan, B. \& Green, M. (Eds.), Comparative Elite Sport Development: systems, structures and public policy $1^{\mathrm{a}}$, pp. 166-193, Burlington: Elsevier. 